Cahiers de recherches médiévales

\title{
L'Ovide moralisé ou Ovide revisité : de métamorphose en anamorphose
}

\section{Catherine Croizy-Naquet}

\section{(2) OpenEdition \\ 12 Journals}

Édition électronique

URL : https://journals.openedition.org/crm/49

DOI : $10.4000 / \mathrm{crm} .49$

ISSN : 1955-2424

Éditeur

Honoré Champion

Édition imprimée

Date de publication : 10 décembre 2002

ISSN : 1272-9752

Référence électronique

Catherine Croizy-Naquet, "L'Ovide moralisé ou Ovide revisité : de métamorphose en anamorphose », Cahiers de recherches médiévales [En ligne], 9 | 2002, mis en ligne le 05 janvier 2007, consulté le 15 décembre 2022. URL : http://journals.openedition.org/crm/49 ; DOI : https://doi.org/10.4000/crm.49

Ce document a été généré automatiquement le 15 décembre 2022.

Tous droits réservés 


\title{
L'Ovide moralisé ou Ovide revisité : de métamorphose en anamorphose
}

\author{
Catherine Croizy-Naquet
}

$1 \mathrm{Au}$ tournant des $\mathrm{XIII}^{\mathrm{e}}$ et $\mathrm{XIV}^{\mathrm{e}}$ siècles, le succès de l'ovide moralisé est un beau témoignage de l'engouement toujours persistant pour le poète antique ${ }^{1}$ : composé par un auteur anonyme bourguignon ou du Centre-Ouest de la France, l'ouvrage de 72000 octosyllabes est une réécriture des Métamorphoses auxquelles s'ajoutent ici ou là d'autres sources, ovidiennes ou non, latines ou françaises ${ }^{2}$. Cette immense entreprise s'inscrit dans le mouvement des interprétations allégoriques des œuvres de l'Antiquité. Elle s'assigne une relecture des mythes au profit d'une morale chrétienne, chacun étant assorti d'une moralisation ou d'une allégorie. Dans un récit qui court des origines du monde jusqu'à la mort de César et son apothéose, trois des quinze livres sont consacrés à la légende troyenne ${ }^{3}$. Point n'est besoin de souligner la fortune dont celle-ci a pu bénéficier en langue vernaculaire, grâce à Benoît de Sainte-Maure qui l'a initiée en adaptant, dans son Roman de Troie, le De excidio Trojae de Darès le Phrygien ${ }^{4}$. À sa suite, les romans de Troie en prose, isolés ou enserrés dans le moule de l'histoire universelle, l'ont reprise, reformulée et épurée, sans jamais rompre avec le texte de Darès, qui est aux yeux des médiévaux le modèle par excellence de l'historien ${ }^{5}$. Dans le traitement «historique» de la matière troyenne, en dépit des singularités de chaque version, l'Ovide moralisé fait figure d'exception. L'étude du Livre douze, où la méthode de travail du compilateur est la plus apparente, révèle comment l'auteur puise chez le poète et chez Homère pour retrouver, en dehors des sentiers battus, les voies du récit d'origine et pulvériser les bornes d'une tradition qui paraissait fermement établie ${ }^{6}$. Au risque de bouleverser les acquis des «historiens ", cette réécriture qui fait le pari du mythe et de la légende trouve, semble-t-il, sa cohérence et son unité dans le choix du vers : par sa capacité de métamorphose, l'octosyllabe convient en effet au récit troyen comme à l'exposé de ses sens cachés, et réconcilie la fable ${ }^{7}$ - le choix du mot n'est pas innocent avec une vérité non plus immanente, aux prises avec une réalité historique, mais une vérité transcendante, enclose dans une perception chrétienne du monde.

L'Ovide moralisé ou la métamorphose du texte ovidien 
2 Fidèle à Ovide dans la partie troyenne, le versificateur conserve en intégralité la trame narrative du Livre XII. Les Métamorphoses lui fournissent quelques épisodes : le sacrifice d'Iphigénie à Aulis, le premier exploit d'Achille sur le rivage de Troie avec la mort de Cygnus et sa métamorphose en cygne, enfin la mort d'Achille. Le contenu est de même respecté et maints passages témoignent d'une transposition aussi littérale que le permet le transfert d'une langue et d'un contexte de réception à l'autre : calquée sur la source, la description de la maison de Renommée présente les mêmes images et un ordre identique dans la disposition des éléments ${ }^{8}$. L'auteur préserve également tous les registres rencontrés dans son texte de base. De rigueur dans les épopées latines, le réalisme des descriptions de bataille qu'Ovide se plaît à rendre ${ }^{9}$ inspire des scènes d'une rare cruauté dans le récit médiéval par leur aspect clinique, scènes que ne dédaignerait pas un réalisateur de film d'horreur (XII 2381 et ss.).

3 Le respect du texte ovidien ne résiste pas cependant au profond remaniement qu'exerce au plan structurel l'auteur. Alors qu'Ovide fait converger tous ses effets sur les métamorphoses, sans s'écarter jamais de son sujet, l'écrivain médiéval les relègue à l'arrière-plan, voire les sacrifie. Certes, le thème est exposé dès les premiers vers de l'œuvre, mais pour être minimisé - il n'est réservé qu'à la Genèse - et pour être condamné pour sa vacuité (I 72-96) ${ }^{10}$. L'auteur occulte la métamorphose au seul profit de l'union originelle de la forme et de la matière, renversement capital qui infère une posture nouvelle devant la trame narrative. Là où Ovide conjoint une série d'histoires traitées en micro-épopées, cousues entre elles par des fils ténus, là où il intègre des récits qui brisent la linéarité contraignante du fil chronologique, son successeur s'ingénie à restituer toutes les étapes de la partie troyenne, en faisant appel à d'autres sources. De translateur assujetti à un texte initial, il devient un compilateur manipulant des textes à combiner et à incorporer judicieusement dans le tissu du récit premier.

4 Dans le livre XII, l'auteur s'inspire selon toute vraisemblance des gloses mythographiques de son manuscrit, en forme de commentaires «littéraires" ou historiques, et de compléments, à quoi s'adjoignent les Héroïdes d'Ovide. Parmi les sources externes, il évoque, à propos d'un détail, l'Achilleïs de Stace - sans doute dispose-t-il d'une glose -; il utilise Homers (v. 1317), en réalité Baebius Italicus et son Ilias Latina; il recourt enfin à la tradition de la légende troyenne profilée par Darès puis Benoît de Sainte-Maure ${ }^{11}$. Il fait donc feu de tout bois, sans préciser toutefois de manière systématique ni l'auteur concerné ni l'étendue de ses emprunts. Pour les longs discours de Pâris et d'Hélène, il adapte avec une grande liberté les épîtres XVI et XVII des Héroïdes, qu'il néglige de nommer, alors qu'il exploite à l'envi la matière de leurs développements psychologiques. En revanche, il cite Stace pour signaler la réclusion d'Achille dans une abeie de nonains sur les ordres de sa mère (v. 100 et ss.). Par le nom d'Homère, l'Ilias Latina est semblablement mentionnée pour de modestes apports, avec les termes estoire, conte, raconter, conter : au port, si com Homers raconte (v. 1317). Elle n'est plus guère évoquée par la suite, lors même qu'elle se trouve à l'origine des épisodes sur le rapt de Criseïs, celui de Briseïs, la bataille auprès des vaisseaux, la mort de Patrocle et la douleur d'Achille, la commande de nouvelles armes pour ce dernier et leur description, la bataille sur les bord du Xanthe, enfin la mort d'Hector ${ }^{12}$. L'adaptateur, il est vrai, use librement de l'œuvre latine, soit qu'il la résume, soit qu'il délaisse la fin du premier livre et les treize suivants, sept cents vers environ, pour reprendre en l'abrégeant ou en s'y alignant la translation du quinzième livre, soit qu'il la complète avec l'ouvrage de Darès. Il colmate les manques narratifs et les brèches temporelles, en 
reliant l'enlèvement d'Hélène au sacrifice d'Iphigénie par deux extraits du De Excidio Trojae de Darès : la poursuite des Dioscures - changés en astres et non plus disparus en mer -, et l'appel d'Agamemnon aux chefs grecs ${ }^{13}$. Alors que son contenu diverge, seule la description des armes d'Achille s'ouvre de nouveau sur un renvoi à la source : si com tesmoigne (vv. 1639-3640). Une certaine désinvolture préside donc à la nomination des sources. Soucieux de faire œuvre de mythographe complet, l'auteur s'efforce avant tout de les nouer harmonieusement à sa trame originelle. Cette pratique explique aussi la présence, à des fins de clarté, d'additions minimes, de provenance incertaine, reprises aux gloses de son manuscrit; d'autres développements adventices, tels le récit de la folie simulée d'Ulysse, ne sont que des prolepses du Livre XIII ${ }^{14}$.

Complexe mais au fond très organisée, la compilation est le moyen de configurer dans son entier la chronologie de l'histoire troyenne, d'en reformuler le cycle et la somme, dans l'esprit d'une époque qui voit prévaloir les synthèses issues du renouvellement des matières romanesque et épique. Si les récits rattachés à la trame initiale risquent de nuire au cours événementiel, la cohésion de l'ensemble est assurée par une claire progression en trois épisodes qui débordent le cadre du seul livre XII, les causes de la guerre, les préparatifs et le conflit ${ }^{15}$.

6 Un obstacle peut-être plus difficile à surmonter est la présence régulière des allégories qui interrompent le cours du récit, d'autant plus nombreuses que se sont multipliées les fables ${ }^{16}$. Occupant environ onze cents vers sur les 3822 que compte le Livre XII, d'origine floue comme l'a montré J.-Y. Tilliette ${ }^{17}$, elles s'enchâssent dans les récits dont elles démultiplient la signifiance: au lieu d'en suspendre la progression, elles l'encadrent et l'interprètent ${ }^{18}$. Au demeurant, l'auteur se définit toujours comme celui qui décrypte la lettre du texte : affleurent les termes raison, savoir, entendement, sens, les syntagmes entendre, retraire, deviser, faire a savoir, gloser autrement, ce que signifie la fable ou l'allegorie, faire aparissable le mistere de ceste fable ${ }^{19} . .$. Le dédoublement du récit païen en une vision chrétienne de la légende s'exerce continûment et tisse de l'un à l'autre de subtiles interférences qui dotent le premier de sens, et font de la seconde le prisme unique de la réception du mythe ${ }^{20}$. Dans l'ensemble de l'Ovide moralisé, les allégories ont pour fondement le dogme de la religion révélée et pour figure centrale le Christ. En profonde symbiose avec une époque où le Christocentrisme est prégnant ${ }^{21}$, l'auteur vénère le fils de Dieu fait homme, dont il donne une sorte de biographie et qu'il dépeint dans sa souffrance. Certaines allégories déroulent le cours de son existence, la huitième par exemple (XII 2881 et ss.) qui évoque l'Annonciation, la naissance de Jésus, sa Passion et sa Résurrection. D'autres s'attardent davantage sur tel ou tel épisode, le moment de l'Incarnation, décrit avec précision et sous des approches variées, jouissant d'un intérêt exceptionnel, parce qu'au plan moral, il a fondé le thème de l'Imitation du Christ, au cœur de la très influente sensibilité franciscaine ${ }^{22}$, et qu'au plan évangélique, il marque la fracture entre un avant et un après la Révélation, entre le temps de la fable, préfiguration des événements, et celui de l'allégorie, énoncé du dogme chrétien et de ses implications spirituelles et morales.

7 Comme l'a démontré M.-R. Jung dans son étude du Livre $\mathrm{XI}^{23}$, les allégories ont la structure du sermon; en totalité ou en partie, elles reposent, dans une habile répartition, sur le récit évangélique focalisé sur la figure du Christ, l'exposé dogmatique et la morale. Dans la septième allégorie, le sacrifice qu'Achille fait d'une génisse à Pallas induit le récit évangélique de la Cène (XII 2110-2113), suivi par le souvenir de la Passion et le rappel de la mission des vrais apôtres, yvre et embeü d'amour (XII 2139 et ss.). Un 
axe temporel traverse sans cesse les allégories, qui oppose les temps précédant et suivant l'Incarnation et que double ou croise un manichéisme systématique : la liste inépuisable des vices (XII 2223-2955) génère par antithèse celle des vertus (XII 2955 et ss.), assimilées et prônées par les religieux, qu'il importe, à l'exemple du Christ, de pratiquer, de même que les visages des mauvais dont celui, emblématique, de Satan, $l i$ traitres, li envieuz, li cuivers, li malicieus (XII 2891-2892) sont, en corollaire, les contraires des bons, les serviteurs de Dieu. Cette esquisse diachronique est l'occasion de représenter la scène du péché originel où s'origine cette fracture irrémédiable, et de retracer la conduite des hommes et les châtiments qu'elle a engendrés, avec le bref rappel des sorts de Sodome et de Gomorrhe puis de l'Égypte (XII 1988 et ss.).

8 Comme dans les autres livres, les allégories se rattachent toujours à la fable ovidienne. Dans la première, qui dénonce la vaine quête des plaisirs, Hélène est la vaine delice (XII 873) et Junon la convoitise (XII 851). Dans la deuxième, la jalousie de Ménélas à l'égard de sa femme est celle de Dieu envers l'âme qu'il veut sauver. Et la vengeance du mari bafoué qui convoque l'armée des Grecs est rédupliquée par celle de Dieu rassemblant autour de lui ses gens, ses especiaulz sergens, les sains predicatours, les mestres et les doctours (XII 1216-1218), [...] ceulz de la table reonde (XII 1242) ${ }^{24}$. À la géographie «réelle» de la fable correspond en outre une géographie morale, le port devenant celui de pardurable vie (XII 1222). La quatrième allégorie fait du sacrifice d'Iphigénie par son père le sacrifice du Christ par Dieu, tandis que la cinquième met en équivalence la Maison de Renommee et l'Écriture omnisciente. La neuvième associe Hercule à Dieu et Nestor à Israël, incité à se convertir. La dixième allégorie interprète les rapts de Briséis puis de Criséîs comme le combat du Bien contre le Mal après le péché originel. Dans la onzième allégorie, Patrocle annonce les saints et les prophètes, et sa mort préfigure leur martyre. La description des armes d'Achille engendre la métaphore filée de la douzième allégorie, à travers le portrait du Christ en armure de Dieu. L'allégorie qui clôt le recueil fait de Polyxène l'image de l'Église sacrifiée.

9 En règle générale, le verbe estre, la préposition com (XII 2034, 2040, 2900) ou des formules plus étoffées (XII 2917-2918) établissent d'entrée l'équivalence. Mais il est parfois nécessaire d'attendre avant de voir un lien s'établir. L'analogie entre Iphigénie et le Christ n'intervient qu'après un long discours sur les saints convertis après une vie dissipée, dont saint Paul, li devins de grant savoir (XII 1528), cil qui de Dieu fu eslis (XII 1529), li apostres qui descrist/De l'avenement antecrist (XII 1539-1540). Dans un rappel d'une allégorie du Livre III, la «métamorphose» de saint Paul, qui incarne l'Église militante ${ }^{25}$, prend son sens avec l'évocation subséquente de la venue du Christ, le prestre [...] qui nous fist pont et planche et voie (XII 1573-1574). Qu'Iphigénie soit dans cette allégorie une figure christique est sans doute faire bon marché de l'action du Christ dans le monde et des actes et intérêts grecs mis en cause. Pourtant, en juxtaposant deux visages de la pureté et de l'innocence, l'analogie éclaire le sacrifice, dans une indicible souffrance, des justes pour sauver les méchants ; la présence de saint Paul qui les «soude » élargit le champ de la théorisation, en passant de la reconnaissance des nécessités du sacrifice à l'attitude militante qu'infère, au sein de son Église, celui du Christ. D'autres rapprochements semblent ou hardis ou artificiels, ainsi le fait d'assimiler dans la cinquième allégorie la maison de Renommée, soumise aux aléas de la nature humaine, à l'Écriture sainte, même si l'une peut annoncer l'autre. Faire du sort de Patrocle une anticipation de celui des saints est mettre sous le boisseau les traits de caractère du héros grec. Mais la logique réside, comme l'a souligné J.-Y. Tilliette ${ }^{26}$, dans 
un mouvement inverse de la démarche ordinaire, qui va de l'allégorie à la fable : par un étoilement à l'infini, la vérité irriguant les digressions religieuses et jaillissant du texte sacré est débusquée et finalisée dans les moindres replis de la fable.

L'existence d'une grille unique explique que les allégories forment un tout homogène qui puisse exister de façon autonome. De l'une à l'autre se produisent des jeux d'échos sémantiques au moyen de mots clés et la reprise de schémas syntaxiques qui font la part belle à la paratax ${ }^{27}$. De l'une à l'autre résonne une même tonalité non dénuée de mysticisme, nuancée par des amplifications et des ajouts « historiques» ou « moraux $»^{28}$, ordonnée autour des tableaux du Christ souffrant et de la peinture d'un Dieu compatissant et magnanime ou d'un Dieu de colère et de vengeance, avec une condamnation virulente des méchants et de leurs vices. Sans être un cours d'histoire sainte, les allégories élaborent et promeuvent une morale de vie, et lui associent une assise spirituelle empreinte de l'humanité du Christ. Ce programme est exécuté à l'échelle de chacune, par le procédé de l'élargissement à partir d'un thème donné, et à l'échelle de l'ensemble, de façon à former une chaîne dont chaque maillon allégorique complète l'autre au rythme du ressassement et du dépassement. Au fur et à mesure du Livre XII, elles vont ainsi en crescendo, de la première d'un ton uniment déploratif à connotation fortement morale, à la dernière qui, intervenant après la mort de Polyxène, est dédiée, après un retour sur la naissance et la Passion du Christ, véritable leitmotiv dans le Livre XII, à la sainte Église : Polixene, la bien aprise/C'est la sainte ame, ou sainte Yglise... (XII 4707-4708), de manière à proclamer sa victoire annoncée sur le monde et sur le mal.

11 L'Ovide moralisé associe donc deux histoires parallèles, deux scénarios à la fois autonomes et complémentaires. L'unité des fables tient pour l'essentiel à la chronologie qui montre de l'une à l'autre la continuité. Celle des allégories réside dans l'exposé du dogme chrétien et son apologie constante, ainsi que dans les exhortations à se bien conduire. L'assemblage de ces modes d'écriture sur le principe de l'alternance engendre, malgré une composition fragmentée, une vision synoptique de la légende troyenne, dont la cohérence est indissolublement rivée à la démarche interprétative menée en parallèle. En dépit des connotations négatives que le terme peut receler (XII 2517, 2524), l'auteur peut à bon droit revendiquer pour ses récits le statut de fable, envisageant même celui que revêt maintes fois l'Écriture sainte :

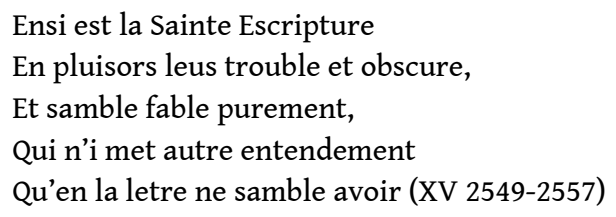

Se conformant au processus allégorique, la fable troyenne préserve son exclusivité et conquiert sa légitimité, car [...] sous la fable gist couverte/ la sentence plus profitable (XV 2536-2537). Une telle «métamorphose » entérine le triomphe de la fable sur l'histoire et marque la revanche du poète sur l'historien.

Sous le voile des fables troyennes à la manière d'Ovide

13 L'auteur de l'Ovide moralisé rompt de manière éclatante avec la tradition «historique ", accréditée en français par Benoît de Sainte-Maure, sous l'autorité et le patronage de Darès. Dans son prologue, le clerc tourangeau clame haut et fort la supériorité du De Excidio Trojae sur l'œuvre d'Homère qui mêle les dieux aux conflits des hommes ${ }^{29}$. Sous la forme d'un palimpseste subtilement recomposé, l'auteur de l'Ovide moralisé détourne, 
dans un passage justement célèbre ${ }^{30}$, l'argumentation de Benoît dont il loue les qualités littéraires, jugeant qu'il est un bons rimourres/cortois parliers et biaus faigtierres (XII 1719-1720) - compliment non négligeable de la part d'un auteur fort sensible à la littérarité de son texte-, mais dénonçant en lui le piètre translateur (XII 1722-1723). Rendant hommage à Homère et Darès, il leur accorde à tous deux la vérité de la matire, bien qu'ils empruntent des voies différentes: Darès dist plus prolixement [...] le demenement, tandis qu'Homère parle par metaphore ${ }^{31}$. Il ignore, ce disant, le grief majeur de Benoît concernant le rôle des dieux et des déesses, sans doute à dessein car, dans sa transposition de l'Ilias latina, il fait intervenir ceux qu'il juge indispensables à l'intelligence du récit ${ }^{32}$.

14 Par cette attaque en règle, l'auteur de l'Ovide moralisé met en échec toute tentative d'historicité et de rationalisation sous l'influence de l'évhémérisme, pour revenir, dans une belle réhabilitation d'Homère, au fabuleux, c'est-à-dire au mythe, quand bien même il s'autorise parfois des emprunts à Benoît ${ }^{33}$. Il ne nie pas du reste la diversité de ses références: la mort d'Achille est même l'occasion de confronter les sources historiques et légendaires, Darès, Benoît et Dictys d'un côté, Ovide de l'autre. Non sans malice, il refuse de prendre parti et laisse au lecteur le soin de trancher. Après le vibrant plaidoyer en faveur d'Homère et par conséquent d'Ovide et de la légende, son apparente neutralité s'explique par le souci intradiégétique de raccorder le récit à sa source première, les Métamorphoses, dont il reprend la traduction. Mais elle est aussi le moyen d'éluder un débat de fond sur les enjeux de l'historicisation de la légende ${ }^{34}$. Après avoir défendu la vérité profonde et secrète des fables, la mise en valeur d'une conscience historique qui leur confère une vérité immédiate ne pouvait, aux yeux de l'auteur, que menacer son entreprise et son pari d'éradiquer la toute-puissance de l'histoire au nom d'une vérité supérieure. Plus le texte en effet est légendaire, plus il se prête à la moralisation; plus il est historique, moins il autorise une lecture symbolique et la révélation d'une vérité transcendante. Si le choix d'Ovide autorise à restituer le vrai visage de la légende, il s'accompagne donc, grâce aux exigences d'élucidation qu'elle suscite, d'un éclairage nouveau qui la dote d'une profondeur et d'un sérieux dont elle se voyait privée dans la conscience médiévale et qui la pare des vertus et des prestiges d'un sens mystérieux à pénétrer.

Dans cette démarche volontairement anhistorique, l'auteur se distingue davantage encore des successeurs de Benoît, avec qui il partageait au moins la pratique du vers et l'usage de procédés épico-romanesques. À l'initiative des prosateurs qui mettent le Roman de Troie en prose ou bien des traducteurs de Darès, la légende troyenne est versée dans le cours de l'histoire, saisie dans son sens littéral et expurgée de toute formulation ambiguë de doctrine ou de spéculation, de toute extravagance provenant de la superstition ou du travail poétique ${ }^{35}$. Le cadre de l'histoire universelle dans lequel elle est parfois coulée reflète au mieux le nouveau contrat engagé avec le temps. L'auteur de l'Histoire ancienne jusqu'à César conjoint ainsi histoire païenne et histoire biblique et, au sein de l'histoire païenne, légende troyenne et histoire romaine ${ }^{36}$. Il rétablit le cours de l'histoire humaine, qu'il projette sur un axe diachronique prenant racine à la Genèse. Il fait se confondre temps historique et temps mythologique, et annihile leurs singularités respectives, au nom d'un rapport au réel qui fonde la vérité historique et se matérialise par la mise à jour de causalités établies dans l'immanence des faits. Il émaille ses récits de moralisations en vers, hormis ceux qui concernent la matière "antique», la geste troyenne singulièrement, afin d'obérer tout 
rapprochement entre les prestigieux romans en vers octosyllabiques et sa propre version, sèche et dépouillée, et de s'ancrer plus encore dans une lecture historique des événements. Ailleurs, souvent réduites à des principes de morale commune, les moralisations portent sur une étude des comportements qui détermine l'évolution des faits et qu'elles visent à réformer. Linéarité constante, stricte diachronie, nivellement de la légende, tout conspire à ramener l'histoire à sa seule dimension humaine et s'oppose à un quelconque verrouillage herméneutique ${ }^{37}$.

16 Par contraste, le poète de l'ovide moralisé repousse la tradition historique de la légende médiévale et travaille à renouer avec la version originelle, en jouant Ovide contre Darès. Ovide lui fournit la trame du récit et les éléments fabuleux qui instillent les ambiguïtés d'une étrangeté familière, et il lui transmet le sens de l'efficacité poétique, avec l'usage du vers et sa capacité infinie à accéder aux zones d'ombre, au sein des êtres et des choses. L'auteur ne rend pourtant guère hommage au génie poétique de son prédécesseur latin. La légende resurgit dans sa pureté, mais dans une langue autre, avec un vers repensé en fonction du lien de la fable à la morale : le vers se mue en un laboratoire où se produit une perpétuelle anamorphose qui, selon les points de vue, dévoile les métamorphoses en allégorie de la fable débarrassée de sa frivolité et la contamination de l'allégorie par la fable. La réhabilitation d'Ovide tient tout entière au projet central d'exposer/d'imposer une pensée morale et mystique, conférant à l'écrivain, outre le statut de conteur de fables, celui de philosophe et de prédicateur.

Dans la tradition médiévale troyenne, le choix du vers et de la fable bouleverse à l'évidence les normes tacitement établies. Les prédécesseurs en prose de l'auteur se sont évertués à dénoncer la collusion du vers et de la fable dans leurs prologues et épilogues, arguant d'un alliage incompatible avec l'expression de la vérité. Si certains se contentent d'exposer leur dire vrai par la prose, d'autres y joignent une condamnation virulente et parfois méprisante des menestriers ${ }^{38}$. Loin de réfuter ces principes, l'auteur de l'Ovide moralisé s'acharne à son tour contre les vaines fictions en les cantonnant dans le champ de l'idolâtrie et récuse le travail du vers à des fins futiles. Aussi condamne-t-il les poètes qui créent par la fontaine de Poesie (V 2689-2697) et louet-il ceux qui, à son exemple, traduisent avec talent les fables pour lire en elles la vérité, à l'aune du texte révélé qui l'offre d'emblée (XV 2546-2556). Extrait de la mouvance strictement historique où il était déclassé, le vers est racheté, ainsi que la fable qui lui est intrinsèquement liée, par le mécanisme de l'allégorisation. Dans la quête du sens, il s'avère l'instrument adéquat pour métamorphoser la fable en vérité, et il est d'autant plus approprié et efficace qu'il est apte, par son potentiel créatif, à frapper l'imagination des auditeurs/lecteurs : qui sait bien raconter sait bien exhiber la letre du récit. Par sa richesse, par sa facture ciselée avec soin, par les correspondances qu'il induit au moyen des consonances et des échos intratextuels, par le «feuilleté » que provoque l'étagement des strates narratives et moralisatrices, il est le lieu où s'accomplit l'alliance de la fable et de l'allégorie, le lieu où se révèlent leurs secrètes ressemblances et se dévoile leur complémentarité consubstantielle.

La vocation du vers pour le récit s'illustre dans la récriture de la légende ovidienne à la lumière de la littérature médiévale. La démarche du poète n'est en ce sens guère novatrice. Benoît de Sainte-Maure a de même renouvelé la matière antique selon les valeurs du temps de la composition, courtoises en particulier, mais son successeur reste libre de tout assujettissement, comme l'illustre, au commencement du Livre XII, l'épisode des amours d'Hélène et de Pâris. Il s'inspire, on l'a dit, des Hérö̈des XVI et XVII 
qu'il transforme en deux longs discours lors d'un dialogue qui réunit les héros, l'enlèvement d'Hélène prenant tout son sens dans le cadre plus large du jugement de Pâris (XI, 1832-2394). Des Héroïdes, il conserve le schéma d'ensemble et les arguments employés, dont le fait qu'Hélène a prémédité de suivre le prince troyen. Prononcée par Pâris, l'épître XVI est toutefois incomplètement reproduite et très abrégée, hormis quelques passages transposés de façon quasi littérale, alors que la suivante, la réponse d'Hélène, est plus fidèle à l'original. L'auteur compense absences et résumés, en entrelaçant aux discours des éléments de l'amour courtois: topoi et motifs, tels la souffrance amoureuse, héritée du reste d'Ovide mais mesurée à l'aune du discours romanesque, ou le devoir d'Amour repensé en termes féodaux (XII 36)-, rimes qui thématisent scènes et situations de la littérature vernaculaire, par exemple ces vers :

Pour vostre amour passai je mer,

Si m'en devez mieus, dame, amer

Quant fiance ai en vostre amour... (XII 129-131)

19 Les formules participent aussi à la coloration courtoise des propos tenus, qu'elles apparaissent en prélude aux discours dans la peinture d'Amour et de sa loi (XII 92-94) ou parmi les arguments choisis (XII 245-248).

Mais là s'arrêtent les influences, l'auteur évidant de son contenu le fondement de l'éthique courtoise. Dans la mouvance ovidienne, il s'écarte de Benoît qui, développant la trame ténue de Darès, faisait des amours d'Hélène et de Pâris, un modèle achevé de perfection amoureuse ${ }^{39}$. À un récit dithyrambique du rapt, aboutissement légitime de la rencontre entre deux êtres parfaits, il substitue une approche beaucoup plus réaliste où l'amour est objet de marchandage et se monnaye en termes de possessions et d'avantages matériels, où surtout il est la conjonction de deux désirs charnels. Il est question de la part de Pâris ou du besier ou de la chose (v. 172), de vostre lit le deduit [...] et le delit (XII 243-244) et de l'incompatibilité entre chasteté et beauté ; Hélène qualifie son engagement amoureux comme folie et putage (v. 381), tout en concédant que le delis lui plairoit (XII 609). Une telle peinture de l'amour, dont l'auteur décrit avec complaisance les délices, traquant comme chez Ovide les tourments du désir plutôt que ceux du sentiment, montre le clivage très net qui s'exerce avec la représentation "romantique » de Benoît.

21 L'usage distancié et peut-être ironique des schèmes courtois où l'euphémisme règne en maitre contribue paradoxalement à la renaissance de la version originelle; les analogies implicites entre un idéal et sa version dégradée accusent les traits de caractère des deux personnages. Hélène pâtit de ce traitement ${ }^{40}$. Décalque de l'Héroïde XVII, son discours est un chef d'œuvre de manipulation, une leçon de stratégie amoureuse et matrimoniale où prime l'intérêt. Il est conforté en ce sens par l'ajout d'une conclusion à l'entretien, empruntée à Darès et dans laquelle est fixé le point ou le leu ou la bele seroit ravie (XII 740-741), et par la scène de l'enlèvement où l'héroïne excelle en feintise et en perversité (XII 776-783). Magnifiée chez Benoît par l'amour voluptueux et exaltant qui l'unit à Pâris, elle est dégradée en femme fatale, cynique, calculatrice, redoutable par sa rouerie. La métamorphose de l'Hélène médiévale des origines en une Hélène qui doit bien davantage à la légende antique, en une pilleuse, une bourrasque $d u$ monde $e^{41}$ guidée par ses seuls instincts, tient moins à une volonté de démystifier l'idéal courtois qu'à l'allégorie que le poète greffe à l'épisode: pour incarner la vaine delice, le visage d'Hélène se devait d'être modifié. 
22 Le travail de réécriture s'exerce dans maints autres passages. Parmi eux, certains privilégient, semble-t-il, l'aspect purement esthétique. La bataille à l'occasion du banquet qui se déploie, pour atteindre les sommets de l'horreur, en un tableau spectaculaire et hyperréaliste, prend son sens grâce à l'allégorie qui la clôt : la violence, agressivement picturale, est à lire comme le symbole de la méchanceté de l'Homme, déclinée en une longue litanie de défauts (XII 2922 et ss.). D’autres passages sont traités sur un mode différent: récurrent dans les littératures latine et médiévale, le motif consacré de la tempête matérialise la colère des dieux contre les Grecs, mais l'allégorie en déplace la signifiance, en le resémantisant: La tempeste qui la mer trouble/C'est pechié, qui le mont tant trouble... (XII 1547-1548). Sur le modèle du planctus, la très belle déploration qui accueille la mort d'Hector (XII 3978 et ss.) est un florilège de réminiscences du Roman de Troie où elle se déployait en plusieurs prises de parole ${ }^{42}$. Elle n'entretient aucun lien apparent avec l'allégorie subséquente dont le thème est la confrontation du Bien et du Mal et la naissance du Christ, armure de Dieu et détenteur du savoir, pour restaurer l'umain lignage (XII 4241) et jeter les mauvais en enfer. Mais peut-être accroît-t-elle la tonalité dramatique de l'épisode et prépare-t-elle la leçon à venir: la mort d'Hector, ou la victoire du Mal sur le Bien, montre la nécessité d'un sacrifié médiatisant l'intervention divine, seule qualifiée pour remédier aux péchés des hommes.

Dans les fables domine donc un souci constant de la forme, le vers épousant la diversité des registres et des tons, entre lyrisme et trivialité, entre grâce et vulgarité, afin de peindre dans leur pleine épaisseur la diversité des êtres et des situations. La force de ces fables, dont le vers accentue le légendaire, reste cependant lettre morte, si les allégories leur sont soustraites. Le jeu de montage dual et le sertissage de l'allégorie dans la fable recyclent et subliment la légende troyenne. Les pièces allégoriques décryptent la fable par l'explication, mais plus encore par un usage du vers qui résonne en écho au vers narratif. Plus que les fables, elles sont marquées au coin du lyrisme né de la ferveur d'une âme engagée. Créant des litanies, la cadence de l'octosyllabe, les consonances parfaites du rythme et de la rime que favorisent les annominatio et les multiples anaphores aboutissent à une sorte de chant liturgique. Cette impression est avivée par l'exacte reprise de vers qui forment des refrains : le distique C'est par les sains predicators,/Par les mestres, par les doctors ponctue par exemple la deuxième allégorie, épousant les sinuosités et les infléchissements du sermon ${ }^{43}$. Par contraste avec la fable, la prolifération des termes abstraits illustre le rôle premier de l'allégorie : éclairer, par le biais de l'analogie, la vérité sur le monde. Mais la présence incessante d'images, de métaphores très vives, de comparaisons frappantes, nourrit le discours d'une suggestivité qu'interdit le seul mode de l'explication philosophico-théologique ${ }^{44}$. La peinture de la morale en termes de géographie, celle de la Vierge en jeune femme pure et chaste et l'allusion récurrente à sa maternité, celle du Christ tour à tour semeur et laboureur, soldat, armure, prêtre, port, étoile de mer, roche, champ, époux de notre Église ${ }^{45}$... humanisent singulièrement le propos, mettant en abyme et reproduisant au vif de la langue l'idée force de toutes les allégories, l'incarnation du fils de Dieu. La démonstration didactique qui sous-tend ces sermons est commuée en un véritable poème à la gloire du Christ.

En rompant avec la tradition médiévale pour revenir, dans l'ignorance des motifs platoniciens et pythagoriciens qui l'ont infléchie, au cœur de la légende troyenne grâce à Ovide, l'auteur de l'Ovide moralisé courait, malgré l'insertion des allégories, le risque 
d'une plate adaptation. Mais l'usage du vers vernaculaire, à la fois souple et polymorphe, prévient tout plagiat. Par l'unité des procédures qu'il engage, par sa capacité à saisir le temps de la fable et celui de l'allégorie et à les harmoniser dans la cohérence des mots et des figures, le vers dont sont exploitées les potentialités figuratives et expressives transcende la linéarité événementielle du récit et prépare de manière souterraine la soudure avec la démonstration allégorique. Le poète fait de cette forme une perpétuelle anamorphose ; par sa plasticité, il est tantôt le réceptacle où se clôt le sens à dévoiler et méditer, tantôt l'écrin où le monde se donne en représentation, où s'opère la hiérarchisation des temps, où la légende finit par rejoindre l'histoire. Mais si chez Ovide, le passage du temps mythologique au temps historique se fait sans solution de continuité, il se produit dans l'ovide moralisé par le processus allégorique qui dédouble, dans une saisie synchronique, fable et allégorie sous l'autorité du texte sacré, emblème de l'éternité divine, et qui dédouble légende et histoire dans une perspective diachronique, avec le moment-clé de l'Incarnation où le Christ rejoint le temps des hommes et celui de l'histoire. Tout comme il est à la charnière de la fable et de l'allégorie qu'il noue en une entité unique, le vers enchaîne la légende à l'histoire et les unit dans le plein acquiescement à leurs statuts et enjeux respectifs.

\section{NOTES}

1.Ovide moralisé, poème du commencement du XIV siècle, éd. C. de Boer (avec la collaboration pour certaines parties de M.G. de Boer et J. Th. M. Van T Sant), Amsterdam, Müller, 5 vol., 1915-1936, réimpr. Vaduz, Wohlwend, 1988. Ovide, Les Métamorphoses, t. 1, éd. et trad. G. Lafaye, Paris, Les Belles Lettres, 1969 (1ère édition, 1928) ; trad. G. Lafaye, présentation J.-P. Néraudau, Paris, Gallimard, Folio classique, 1992.

2.D’Ovide sont utilisées les Héroïdes, éd. H. Bornecque, traduit par M. Prévost, Paris, Les Belles Lettres, 1965 (1ère édition 1928).

3.Dans l'Ovide moralisé, la partie troyenne couvre les livres XI à XIII. Voir la présentation de M.-R. Jung, La légende de Troie en France au Moyen Âge. Analyse des versions françaises et bibliographie raisonnée des manuscrits, Bâle-Tübingen, Francke, Romanica Helvetica, 114, 1996, pp. 621-628.

4.Benoît de Sainte-Maure, Le Roman de Troie, éd. L. Constans, Paris, Firmin Didot, S.A.T.F., 6 vol., 1904-1912 ; éd. et trad. E. Baumgartner, F. Vielliard, Paris, Le Livre de Poche, Lettres gothiques, 1998. Darès, De excidio Trojae historia, éd. F. Meister, Lipsiae, Teubner, 1873 et Dictys, Ephemeridos belli Troiani libri a Lucio Septimio ex Graeco in latinum sermonem translati, éd. W. Eisenhut, Leipzig, Teubner, 1973. Ces deux textes se lisent dans Récits inédits sur la guerre de Troie, traduits et commentés par G. Frye, Paris, Les Belles Lettres, La roue à Livres, 1998.

5.Cf. B. Wooledge, «La légende de Troie et les débuts de la prose française », Mélanges de linguistique et de littérature romanes offerts à M. Roques, Paris, 1953, t. II, pp. 315-325. 
6.Sous le nom d'Homère, il faut voir, comme l'observe l'éditeur de l'Ovide moralisé, Baebius Italicus, Ilias latina, éd. Vollmer, Poetae latini minores, Teubner, Leipzig, 1913. 7.Sur le sens de ce terme, des origines au Moyen Âge, voir P. Demats, Fabula. Trois études de mythographie antique et médiévale, Genève, Droz, Publications romanes et françaises, 1973.

8.XII 1588 et ss.; Métamorphoses, XII 39-63.

9.XII 210-462. Sur ce réalisme et son origine, voir éd. cit., note p. 570

10.Cf. l'analyse de P. Demats, op. cit., p. 169 et ss.

11.Sur les sources, cf. P. Demats, op. cit., p. 63 et ss. ; M.-R. Jung, op. cit., p. 622.

12.Cf. XII 3226-4183, où l'auteur « recompose » l'Ilias latina, l'ensemble étant ponctué par les allégories.

13.Cf. Darès, op. cit., XI.

14.Voir P. Demats, op. cit., p. 92.

15.Progression mise en valeur par P. Demats, op. cit., p. 92 et ss.

16.Le livre XII comporte treize allégories : 827-877, 1205-1316 (deux allégories se suivent), 1511-1582, 1657-1708, 1925-2044, 2084-2155, 2881-3034, 3139-3225, 2247-3423, 3527-3582, 4184-4304, 4703-4794.

17.J.-Y. Tilliette, «L'écriture et sa métaphore. Remarques sur l'Ovide moralisé », Ensi firent li ancessor, Mélanges de philologie médiévale offerts à M.-R. Jung, dir. L. Rossi, Ch. Jacob-Hugon, U. Bähler, Alessandria, Edizioni dell'Orso, 1996, vol. II, pp. 543-558. 18.Sur les quatre interprétations possibles des fables d'Ovide (physique, historique, morale et religieuse), voir la typologie établie par M. Possamaï, «Les Dieux d'Ovide «moralisés » dans un poème du commencement du XIV ${ }^{e}$ siècle », Bien dire et bien aprandre, 12, 1994, pp. 203-214.

19.Voir par exemple XII 827, 1205, 1660, 2027, 2028, 2084, 2085, 2881-2882, 3150...

20.Cf. l'analyse de M.-R. Jung, « Aspects de l'Ovide moralisé », Ovidius redivivus. Von Ovid zu Dante, éd. M. Picone, B. Zimmermann, Stuttgart, M \&P, 1994, pp. 149-172.

21.Sur ce point, consulter par exemple A. Vauchez, La Spiritualité du Moyen Âge occidental. VIII ${ }^{-}$-XIII ${ }^{\text {s }}$ iècle, Paris, Seuil, Points histoire, 1994, p. 140 et ss.

22.La focalisation sur le Christ s'accompagne en effet du thème de l'imitation, développé par les Franciscains, et s'exerce par une dévotion marquée à l'Incarnation et à la Passion. S'il n'est pas certain que l'auteur ait appartenu à l'ordre des frères mineurs (éd. C de Boer, éd. cit., t. 1, introduction, p. 9), au moins est-il établi que son œuvre a rencontré un vif succès dans ce milieu.

23.M.-R. Jung, art. cit., p. 161, 167.

24. Doit-on comprendre qu'il s'agit de la table de la Cène autour de laquelle le Christ a réuni les apôtres? Si tel est le cas, l'auteur accorde crédit à la reconstitution «historique » de la tradition arthurienne qui fait se succéder trois tables, la dernière étant celle du roi Arthur.

25.III 1247-1272. Voir l'analyse de. J.-Y. Tilliette, art. cit., p. 550.

26.J.-Y. Tilliette, art. cit., p. 557.

27.Sur cette pratique, que recommande les artes praedicandi, par association et par analogie typique du sermon ou de la prédication, $\mathrm{cf}$. M. Zink, La prédication en langue romane avant 1300, Paris, Champion, NBMA, 1976, pp. 248-249. Voir aussi M.-R. Jung, art. cit., p. 161 et ss. qui en a fait la démonstration pour le Livre XI. Des réseaux sémantiques se forment autour de deux grands axes : celui de la vengeance de Dieu avec ses causes (le péché décliné dans une longue liste des défauts) et ses modalités (le terme 
Enfer en est le mot-programme) ; celui de la naissance du Christ, sauveur de l'umain lignage (les temps de l'Incarnation et de la Passion sont sans cesse repris). Ces deux axes structurent les allégories, pouvant se nuancer en une opposition Dieu et Diable (XII 1925), et s'ordonnent le plus souvent par le biais de l'association de mots - et d'idées - à partir d'un terme initial, chaque allégorie conservant sa spécificité. Le terme dyable qui ouvre quasiment la sixième allégorie crée une chaîne sémantique du mal, du péché, de l'enfer, de la violence, tandis qu'en contrepoint se développent les termes Dieu, filz de Dieu, Vierge Marie, vengeance, etc.

28.Voir par exemple XII 3139 et ss.

29.L'épisode des amours d'Achille et de Polyxène est résumé du Roman de Troie: cf. Roman de Troie, éd. et trad. E. Baumgartner et F. Vielliard, vv. 45-74.

30. XII 1712-1754 ; cf. P. Demats, op. cit., p. 96 et ss.

31.Présupposé aujourd'hui paradoxal qui laisse entendre que l'auteur disposait d'une version incomplète de l'Ilias latina (plutôt que d'une version plus développée de Darès) : cf. P. Demats, op. cit., p. 97 et ss. ; d'où peut-être l'abrègement drastique que subit l'Ilias latina dans son adaptation française, à moins que l'auteur ne se refuse à un décrochage trop long sur le seul personnage d'Achille qui pourrait menacer la succession et l'équilibre des épisodes.

32.Sur cet aspect, voir P. Demats, op. cit., p. 98.

33.Cf. P. Demats, op. cit., p. 99 ; Roman de Troie, éd. cit., v. 17545 et ss.

34.P. Demats, op. cit., p. 101 et ss.

35.Sur les différentes mises en prose, consulter M.-R. Jung, op. cit., pp. 431-562. Voir aussi les traductions littérales de Darès par Jofroy de Waterford et Jean de Flixecourt : Jean de Flixecourt, The Old French Johannes Translation of the Pseudo-Turpin Chronicle. A critical Edition. Supplement, éd. R.N. Walopole, Berkeley-Los Angeles-London, University of California Press, 1976, pp. 370-385 (manuscrit de Copenhague) et pp. 386-392 (manuscrit de Turin). Voir F. Vielliard, « La traduction du De Excidio Troiae de Darès le Phrygien par Jofroy de Waterford », Bien Dire et bien aprandre, 10, 1992, pp. 185-205 ; «La traduction du De Excidio Troiae de Darès le Phrygien par Jean de Flixecourt », Medieval Codicology, Iconography, Literature and Translation. Studies for Keith Val Sinclair, éd. P. R. Monks et D.D.R. Owen, Leiden-New-Tork-Köln, E.J. Brill, 1994, pp. 284-295.

36. Histoire ancienne jusqu'à César. Édition de la section Genèse (I) par M. J. Coker-Joslin, The Heard Word: A Moralized History. The Genesis Section of the HISTOIRE ANCIENNE in a Text from Saint-Jean d'Acre, University of Mississippi, Romance Monographs, 46, 1986. Édition des sections Assyrie, Thèbes, Le Minotaure, les Amazones, Hercule (III-IV) par M. de Visser-van Terwisga, Histoire ancienne jusqu'à César (Estoires Rogier), Orléans, Paradigme, 1995, t. 1. Édition de la section Troie d'après Darès (V) par M.-R. Jung, La légende de Troie en France au Moyen Âge. Analyse des versions françaises et bibliographie raisonnée des manuscrits, pp. 358-430. Manuscrit B.N.F. fr. 20125 pour les sections Rome I (VII) et Rome II (X-XI): [fo $\left.177 \mathrm{v}^{\circ}-183 \mathrm{r}^{\circ}\right]-\left[\mathrm{f}^{\circ} 258 \mathrm{v}^{\circ}-375 \mathrm{v}^{\circ}\right]$.

37.Voir notre ouvrage, Écrire l'Histoire romaine au début du XIII siècle : l'Histoire ancienne jusqu'à César et les Faits des Romains, Paris, Champion, NBMA, 1999.

38. Voir par exemple l'épilogue du Roman de Troie en prose (manuscrit 1612), [fo 142 a] : « je la translatai en françois et non pas en rime ne par vers ou il covient par fine force avoir maintes menchoignes com font ces menestriers [...] mais par droit conte selonc ce que je trovai sans riens covrir de verité ou de mençoinge demoustrer ». 
39.Sur ce point, voir notre article, «La complainte d'Hélène dans le Roman de Troie (vv. 22920-23011)», Romania, CXI, 1990, pp. 75-91.

40.Sur le personnage d'Hélène au fil du temps, voir J.-L. Backès, Le mythe d'Hélène, Clermont-Ferrand, Éd. Adosa, 1984, réimpr. 1995.

41.Extrait d'un poème de M. Tsvetaieva cité par J.-L. Backès, op. cit., p. 166.

42.Roman de Troie, vv. 16317-16502.

43.XII 1217-1218, XII 1243-1244 ; voir aussi XII 1677-1678 ; 1687-1688...

44.Elles constituent un mode d'écriture à part entière $:$ la douzième allégorie où le Christ est assimilé à l'armure de Dieu en est l'illustration (XII 4184 et ss.).

45.XII 1224 et ss.

\section{AUTEUR}

CATHERINE CROIZY-NAQUET

Université Lille III 